\title{
Why the Co-based 115 compounds are different: The case study of $\operatorname{Gd} M \operatorname{In}_{5}(M=\operatorname{Co}, \operatorname{Rh}, \operatorname{Ir})$
}

\author{
Jorge I. Facio, ${ }^{1,2}$ D. Betancourth, ${ }^{1,2}$ Pablo Pedrazzini, ${ }^{1,2}$ V. F. Correa, ${ }^{1,2}$ V. Vildosola,,${ }^{2,3}$ \\ D. J. García, ${ }^{1,2}$ and Pablo S. Cornaglia ${ }^{1,2}$ \\ ${ }^{1}$ Centro Atómico Bariloche and Instituto Balseiro, CNEA, 8400 Bariloche, Argentina \\ ${ }^{2}$ Consejo Nacional de Investigaciones Científicas y Técnicas (CONICET), Ciudad Autonoma de Buenos Aires, Argentina \\ ${ }^{3}$ Departamento de Materia Condensada, GIyA, CNEA (1650) San Martín, Provincia de Buenos Aires, Argentina
}

(Received 4 November 2014; published 9 January 2015)

\begin{abstract}
The discovery in 2001 of superconductivity in some heavy fermion compounds of the $R M \operatorname{In}_{5}(R=4 f$ or $5 f$ elements; $M=\mathrm{Co}, \mathrm{Rh}$, Ir) family, has triggered an enormous amount of research into understanding the physical origin of superconductivity and its relation with magnetism. Although many properties have been clarified, there are still crucial questions that remain unanswered. One of these questions is the particular role of the transition metal in determining the value of critical superconducting temperature $\left(T_{C}\right)$. In this work, we analyze an interesting regularity that is experimentally observed in this family of compounds, where the lowest Néel temperatures are obtained in the Co-based materials. We focus our analysis on the $\operatorname{Gd} M \operatorname{In}_{5} \operatorname{compounds}$ and perform density-functional-theory-based total-energy calculations to obtain the parameters for the exchange coupling interactions between the magnetic moments located at the $\mathrm{Gd}^{3+}$ ions. Our calculations indicate that the ground state of the three compounds is a $C$-type antiferromagnet determined by the competition between the first- and second-neighbor exchange couplings inside $\mathrm{GdIn}_{3}$ planes and stabilized by the couplings across $M \mathrm{In}_{2}$ planes. We then solve a model with these magnetic interactions using a mean-field approximation and quantum Monte Carlo simulations. The results obtained for the calculated Néel and Curie-Weiss temperatures, the specific heat, and the magnetic susceptibility are in very good agreement with the existent experimental data. Remarkably, we show that the first-neighbor interplane exchange coupling in the Co-based material is much smaller than in the Rh and Ir analogs which leads to a more two-dimensional magnetic behavior in the former. This result explains the observed lower Néel temperature in Co-115 systems and may shed light on the fact that the Co-based 115 superconductors present the highest $T_{C}$.
\end{abstract}

DOI: 10.1103/PhysRevB.91.014409

PACS number(s): 75.50.Ee, 63.20.D-, 71.20.-b, 65.40.De

\section{INTRODUCTION}

The family of compounds $R M \operatorname{In}_{5}(M=\mathrm{Co}, \mathrm{Rh}, \mathrm{Ir})$, where $R$ is a rare earth, presents a rich variety of electronic and magnetic properties ranging from heavy fermion behavior and anomalous superconductivity to complex magnetic states. These properties are closely related to the strong correlations on the $R 4 f$ electrons and to the quasi-two-dimensionality of the Fermi surface. These materials crystallize in a tetragonal structure that can be viewed as alternating $M \mathrm{In}_{2}$ and $R \mathrm{In}_{3}$ planes stacked along the $c$ axis (see Fig. 1), where the role of the transition metal $M$ connecting the $R \operatorname{In}_{3}$ planes is central to determining the stability of the low-temperature phase.

The most puzzling features occur in the Ce-based compounds, which present heavy fermion behavior at $T \lesssim 20 \mathrm{~K}$. Correlation effects induce an enhancement of the electronic specific heat coefficient up to $1000 \mathrm{~mJ} / \mathrm{mol} \mathrm{K}^{2}$ for CeCoIn ${ }_{5}$, which is an ambient pressure superconductor below $T_{C}=$ $2.3 \mathrm{~K}$ [1]. CeIrIn 5 has its superconducting transition at $T_{C}=0.4 \mathrm{~K}$, while $\mathrm{CeRhIn}_{5}$ is an antiferromagnet at ambient pressure with a Néel temperature $T_{N}=3.8 \mathrm{~K}$ [2]. For $P>$ $P_{\text {cr }}=1.77 \mathrm{GPa}$ the antiferromagnetic state of $\mathrm{CeRhIn}_{5}$ is replaced by a superconducting state which coexists with magnetic order [3]. The less studied $\mathrm{PuCoIn}_{5}$ and PuRhIn 5 compounds are heavy fermion superconductors and the highest superconducting temperature is also obtained for the Co-based compound with $T_{C}=2.5 \mathrm{~K}$, while the reported value for PuRhIn $_{5}$ is $T_{C}=1.1 \mathrm{~K}[4,5]$.

The metallic $R M \mathrm{In}_{5}(R=\mathrm{Nd}, \mathrm{Sm}, \mathrm{Gd}, \mathrm{Tb}, \mathrm{Dy}, \mathrm{Ho}$, Er, and $\mathrm{Tm}, M=\mathrm{Co}, \mathrm{Rh}$, Ir) compounds which order antiferromagnetically, show an interesting pattern in their Néel temperatures (see Table I). For a given rare earth, the Rhand Ir-based compounds have similar Néel temperatures while those based on Co order at a temperature 30\%-50\% lower. In this article we address the above-mentioned regularity and, to that aim, we focus our analysis on the magnetic behavior of the $R=$ Gd compounds. The Gd-115 compounds are particularly appealing to study the role of the transition metal $d$ electrons on the magnetism, because of their relative simplicity. In these compounds the $\mathrm{Gd}^{3+}$ ions are expected to be in a $S=7 / 2, L=$ 0 multiplet, and the crystal-field splitting effects are therefore expected to be much smaller than in $\mathrm{Ce}$ and other $L \neq 0$ rare-earth analogs. Moreover, these materials do not show heavy fermion behavior which further simplifies the analysis. A deeper understanding of the behavior of the magnetic 115 compounds when the transition metal $M$ is replaced may help asses the stability of the superconducting state in the Ce115 and $\mathrm{Pu}-115$ compounds. The superconductivity in these materials seems to be deeply associated with the magnetic properties and the highest superconducting temperatures are obtained for the Co-based compounds.

Total-energy calculations of the $\mathrm{Gd} M \mathrm{In}_{5}$ compounds, based on density-functional theory (DFT), indicate a ground state with magnetic moments localized at the $\mathrm{Gd}^{3+}$ ions and allowed us to estimate the strength of the Gd-Gd magnetic interactions. We solved the resulting magnetic model to obtain the magnetic contribution to the specific heat, the magnetic susceptibility, and the Néel and Curie-Weiss temperatures. The excellent agreement obtained with the available experimental data validates our model and the calculated magnetic interaction 


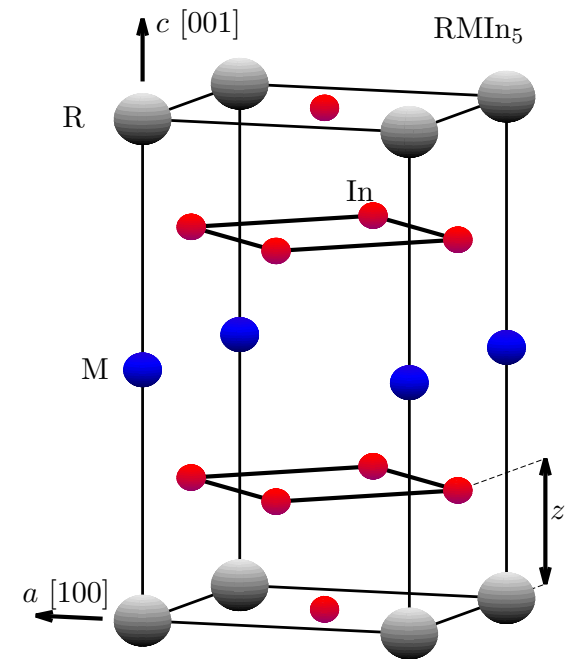

FIG. 1. (Color online) Crystal structure for the $R M \mathrm{In}_{5}$ compounds. The In atoms are represented by red spheres, the transition metal by blue spheres, and the rare earth by gray spheres.

parameters. As we show below, the fact that the $\mathrm{GdCoIn}_{5}$ compound has a lower transition temperature than its $\mathrm{Rh}$ and Ir counterparts can be associated with its strongly suppressed magnetic coupling between $\mathrm{Gd}^{3+}$ ions located at different $\mathrm{GdIn}_{3}$ planes.

The reduced value for the interplane exchange coupling obtained in $\mathrm{GdCoIn}_{5}$ is mainly due to a suppression of the hybridization between the Co $3 d$ and the $\mathrm{Gd}^{3+} 5 d$ orbitals that mediate the interplane Ruderman-Kittel-Kasuya-Yosida (RKKY) interaction between the $\mathrm{Gd}^{3+}$ ion magnetic moments. A toy model considering a single effective $d$ orbital on the transition metal $M$ and the $\mathrm{Gd}^{3+}$ ions is able to qualitatively explain the behavior of the interplane exchange coupling. The parameters for the model were calculated from a Wannier orbital analysis [19] and roughly estimated from the average energy and the total width of the $\mathrm{Co}$ an $\mathrm{Gd}$ bands with dominating $3 d$ and $5 d$ characters, respectively. The results from the two approaches lead to the same conclusions.

The rest of the paper is organized as follows. In Sec. II we determine the magnetic structure and the coupling constants of the magnetic Hamiltonian for the three $\operatorname{Gd} M \mathrm{In}_{5}$ compounds and solve the model in the mean-field approximation and numerically using quantum Monte Carlo (QMC). In Sec. III we summarize our main results and conclusions.

TABLE I. Néel and superconducting transition temperatures for $R M \mathrm{In}_{5}$ compounds at ambient pressure. All temperatures are in $\mathrm{K}$ and bold numbers correspond to the superconducting transition temperature. Superscript letters correspond to references: $a=[1]$, $\mathrm{b}=[6], \mathrm{c}=[7], \mathrm{d}=[8], \mathrm{e}=[9], \mathrm{f}=[10], \mathrm{g}=[4], \mathrm{h}=[11]$, $\mathrm{i}=[12], \mathrm{j}=[13], \mathrm{k}=[14], \mathrm{l}=[15], \mathrm{m}=[16], \mathrm{n}=[5], \mathrm{o}=[17]$, and $\mathrm{p}=[18]$.

\begin{tabular}{lcccccccccc}
\hline \hline$M \backslash R$ & $\mathrm{Ce}$ & $\mathrm{Nd}$ & $\mathrm{Sm}$ & $\mathrm{Gd}$ & $\mathrm{Tb}$ & $\mathrm{Dy}$ & $\mathrm{Ho}$ & $\mathrm{Er}$ & $\mathrm{Tm}$ & $\mathrm{Pu}$ \\
\hline $\mathrm{Co}$ & $\mathbf{2 . 3 ^ { \mathrm { a } }}$ & $8^{\mathrm{b}}$ & $11.9^{\mathrm{c}}$ & $30^{\mathrm{d}}$ & $30.2^{\mathrm{e}}$ & $20^{\mathrm{e}}$ & $10.5^{\mathrm{e}}$ & $<2^{\mathrm{e}}$ & $2.6^{\mathrm{f}}$ & $\mathbf{2 . 5}^{\mathrm{g}}$ \\
$\mathrm{Rh}$ & $3.8^{\mathrm{h}}$ & $11.6^{\mathrm{i}}$ & $15^{\mathrm{j}}$ & $39.9^{\mathrm{k}}$ & $45.5^{\mathrm{l}}$ & $28.1^{\mathrm{m}}$ & $15.8^{\mathrm{m}}$ & $3-4^{\mathrm{k}}$ & $3.6^{\mathrm{k}}$ & $\mathbf{1 . 1}^{\mathrm{n}}$ \\
$\mathrm{Ir}$ & $\mathbf{0 . 4}^{\mathrm{o}}$ & $13.7^{\mathrm{i}}$ & $14.3^{\mathrm{j}}$ & $42^{\mathrm{j}}$ & $41.4^{\mathrm{p}}$ & & & & & \\
\hline \hline
\end{tabular}

\section{MAGNETIC PROPERTIES}

In this section we analyze the magnetic structure of the $\operatorname{Gd} M \mathrm{In}_{5}$ compounds. We propose a simple Hamiltonian to describe their magnetic properties and determine the model parameters through DFT calculations. To describe the temperature dependence of the magnetic properties we first treat the magnetic Hamiltonian in the mean-field approximation which allows a simple interpretation of the experimental data. We then include quantum fluctuations in a simplified model to obtain a quantitative description of the low-temperature $\left(T \lesssim T_{N}\right)$ experimental data.

\section{A. Technical details of the DFT calculations}

The total-energy calculations were performed using the generalized gradient approximation (GGA) of Perdew, Burke, and Ernzerhof for the exchange and correlation functional as implemented in the WIEN2K code [20,21]. A local Coulomb repulsion was included in the $\mathrm{Gd} 4 f$ shell and treated using $\mathrm{GGA}+U$, which is a reasonable approximation for these highly localized states. GGA $+U$ has been also used in previous calculations of compounds of the $R M \mathrm{In}_{5}$ family [22-24]. Due to the localized character of the $4 f$ electrons, the fully localized limit was used for the double counting correction [25]. We described the local Coulomb and exchange interactions with a single effective local repulsion $U_{\text {eff }}=$ $U-J_{H}=6 \mathrm{eV}$ as in bulk $\mathrm{Gd}[26,27]$. Given that the $\mathrm{Gd}^{3+}$ atomic ground state has $L=0$, for simplicity, we have not included spin-orbit interaction in our calculations. The APW + localorbitals method of the WIEN2K code was used for the basis function [20]. For the full optimization of the crystal structures $1200 \mathbf{k}$ points were used in the irreducible Brillouin zone, and the plane wave cutoff $K_{\max }$ was set by fixing $R K_{\max }=8.5$, where $R=2.4$ a.u. is the muffin-tin radii of the In atoms. Regarding the $2 \times 2 \times 2$ supercell totalenergy calculations of the different magnetic configurations, we obtained well-converged results using $440 \mathbf{~ k}$ points and $R K_{\max }=8$. The materials under study in this article crystallize in the tetragonal $\mathrm{HoCoGa}_{5}$ structure ( $P 4 / \mathrm{mmm}$ space group), which is fully defined by the lattice parameters a and c plus one internal coordinate $z$ for one of the In ions (see Fig. 1). The relaxed lattice parameters for the three Gd compounds are presented in Table II.

\section{B. Magnetic structure of the ground state and coupling constants}

We explored different static magnetic configurations for the magnetic moments, which are presented in Fig. 2. The lowest energy configuration for the three Gd compounds is antiferromagnetic (AF3), which corresponds to the measured structure in $\mathrm{GdRhIn}_{5}$ via resonant x-ray diffraction experiments, $\mathrm{NdRhIn}_{5}$ in neutron diffraction experiments, and the inferred structure of DyRhIn ${ }_{5}$ and $\mathrm{HoRhIn}_{5}$ in magnetization experiments $[16,28,29]$. This magnetic configuration is associated with the competition of the first-neighbor $K_{0}$ and the second-neighbor $K_{1}$ antiferromagnetic exchange couplings that lead to ferromagnetic chains in the $\operatorname{GdIn}_{3}$ plane and an antiferromagnetic interplane coupling $K_{2}$ that leads to an antiferromagnetic configuration between $\mathrm{GdIn}_{3}$ planes 
TABLE II. Structural parameters (in $\AA$ ). Superscript letters correspond to references: $\mathrm{a}=[8], \mathrm{b}=[14]$, and $\mathrm{c}=[13]$.

\begin{tabular}{lccc}
\hline \hline & & Experiment & GGA \\
\hline & $\mathrm{a}$ & $4.568(1)^{\mathrm{a}}$ & 4.606 \\
$\operatorname{GdCoIn}_{5}$ & $\mathrm{c}$ & $7.4691(7)^{\mathrm{a}}$ & 7.559 \\
& $z / \mathrm{c}$ & & \\
& $\mathrm{a}$ & $4.606^{\mathrm{b}}$ & 0.3077 \\
& $\mathrm{c}$ & $7.439^{\mathrm{b}}$ & 4.685 \\
$\operatorname{GdRhIn}_{5}$ & $z / \mathrm{c}$ & $0.3025^{\mathrm{b}}$ & 7.555 \\
& $\mathrm{a}$ & $4.622(4)^{\mathrm{c}}$ & 0.3024 \\
& $\mathrm{c}$ & $7.413(8)^{\mathrm{c}}$ & 4.700 \\
$\operatorname{GdIrIn}_{5}$ & $z / \mathrm{c}$ & & 7.545 \\
& & & 0.3019 \\
\hline \hline
\end{tabular}

$[16,28,29]$. The total energy for each magnetic configuration is presented in Table III.

We assume that the magnetic interaction between the magnetic moments on the $\mathrm{Gd}^{3+}$ ions can be described with
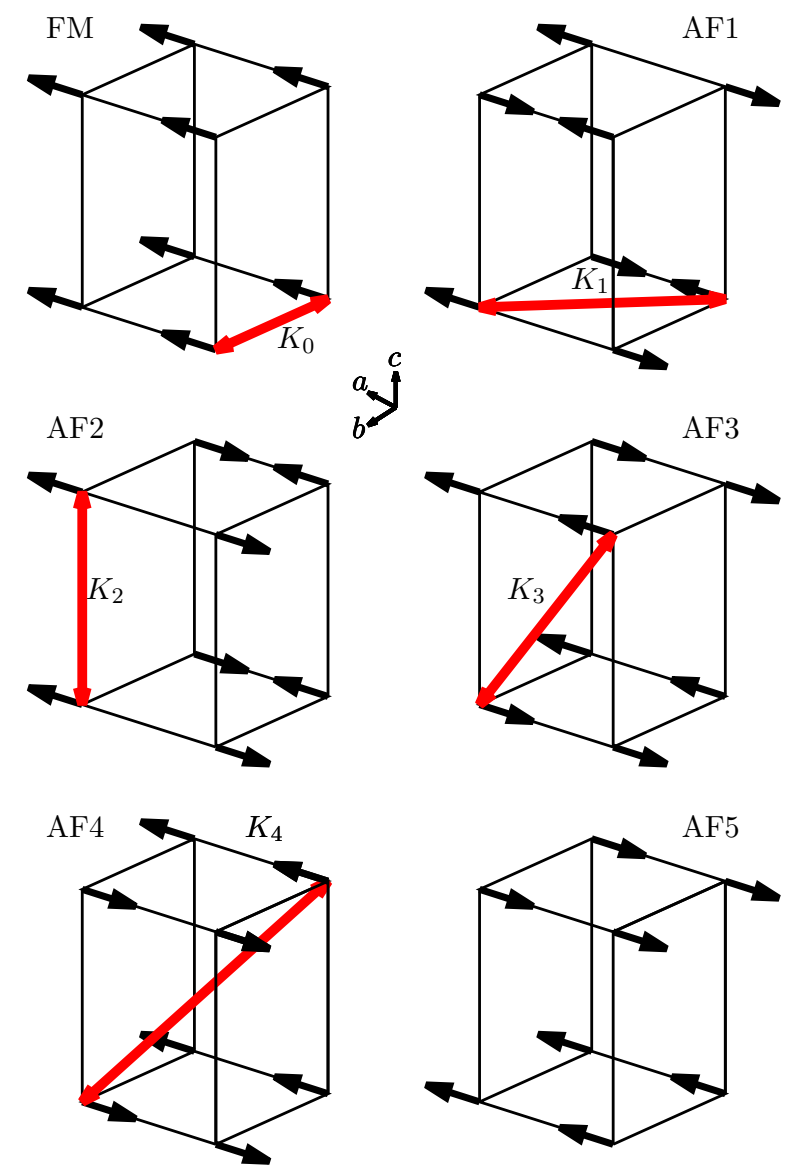

FIG. 2. (Color online) Magnetic configurations proposed to determine the ground state and obtain the exchange coupling parameters. The $\mathrm{Gd}$ atoms are located at the vertices of the rectangular prism and the orientation of their magnetic moments is indicated by black arrows. The red arrows connect a pair of Gd atoms that are magnetically coupled through the exchange coupling parameters $K_{0}$, $K_{1}, K_{2}, K_{3}$, and $K_{4}$, as indicated in the figure.
TABLE III. Relative energy $\Delta E$ (in $\mathrm{K}$ ) with respect to the ground state for the magnetic configurations of Fig. 2.

\begin{tabular}{lccr}
\hline \hline & GdCoIn $_{5}$ & GdRhIn $_{5}$ & GdIrIn $_{5}$ \\
\hline FM & 127 & 145 & 149 \\
AF1 & 62 & 65 & 56 \\
AF2 & 58 & 95 & 74 \\
AF3 & 0 & 0 & 0 \\
AF4 & 23 & 50 & 44 \\
AF5 & 122 & 134 & 128 \\
\hline \hline
\end{tabular}

the Hamiltonian

$$
\mathcal{H}=\sum_{i \neq j} K_{i j} \mathcal{J}_{i} \cdot \mathcal{J}_{j},
$$

where $K_{i j}$ is the exchange coupling between the magnetic moments $\mathcal{J}_{i}$ and $\mathcal{J}_{j}$ and depends on the intraplane and interplane distances between Gd atoms. As indicated in Fig. 2, $K_{i j}$ is equal to $K_{0}$ for nearest neighbors and $K_{1}$ for next-nearest neighbors inside the $\mathrm{GdIn}_{3}$ plane and, correspondingly, to $K_{2}, K_{3}$, and $K_{4}$ for the interplane couplings. The dominating Gd-Gd magnetic exchange interactions are due to a RKKY coupling between the Gd's magnetic moments through exchange coupling with the conduction electrons.

In the absence of an applied magnetic field, the contribution per Gd atom to the total energy due to the magnetic interactions described in Eq. (1) for the different configurations of Fig. 2 is given by

$$
\begin{aligned}
& E_{\mathrm{FM}}^{m} / J^{2}=2 K_{0}+2 K_{1}+K_{2}+4 K_{3}+4 K_{4}, \\
& E_{\mathrm{AF} 1}^{m} / J^{2}=-2 K_{0}+2 K_{1}-K_{2}+4 K_{3}-4 K_{4}, \\
& E_{\mathrm{AF} 2}^{m} / J^{2}=-2 K_{0}+2 K_{1}+K_{2}-4 K_{3}+4 K_{4}, \\
& E_{\mathrm{AF} 3}^{m} / J^{2}=-2 K_{1}-K_{2}+4 K_{4}, \\
& E_{\mathrm{AF} 4}^{m} / J^{2}=-2 K_{1}+K_{2}-4 K_{4}, \\
& E_{\mathrm{AF} 5}^{m} / J^{2}=2 K_{0}+2 K_{1}-K_{2}-4 K_{3}-4 K_{4},
\end{aligned}
$$

where $J=7 / 2$ is the angular momentum of the $\mathrm{Gd}^{3+}$ ion $4 f$ electrons. The energy differences between magnetic configurations calculated from first principles can be combined with Eqs. (2) to obtain the coupling parameters $K_{i}$ solving a system of 5 linear equations. The results for the $K_{i}$ are presented in Table IV and show some remarkable features. On the one hand, the interplane coupling $K_{2}$ is a factor $\sim 3$ smaller in $\mathrm{GdCoIn}_{5}$ than in $\mathrm{GdRhIn}_{5}$ and $\mathrm{GdIrIn}_{5}$, while the other sizable couplings do not change significantly. On the other hand, $K_{3}$ and $K_{4}$ are much smaller than $K_{2}$ so that $K_{2}$ dominates the interplane coupling in the $\mathrm{Rh}$ an $\mathrm{Ir}$ compounds. This implies a more two-dimensional behavior of the magnetism in $\mathrm{GdCoIn}_{5}$ than in $\mathrm{GdRhIn}_{5}$ and $\mathrm{GdIrIn}_{5}$ and, as we see below, explains the lower Néel temperature observed in the Co-based compound.

\section{Toy model for the interplane coupling $K_{2}$}

The exchange couplings calculated in the previous section stem from a RKKY mechanism mediated by the conduction electrons [30]. The $\mathrm{Gd}^{3+} 4 f$ electrons couple with the $\mathrm{Gd}^{3+}$ 
TABLE IV. Calculated exchange couplings (in $\mathrm{K}$ ) and the associated mean-field Curie-Weiss $\theta$ and Néel $T_{N}^{\mathrm{MF}}$ temperatures. $T_{N}^{\mathrm{QMC}}$, Néel temperature calculated using QMC on an effective model (see text). The experimental Néel $T_{N}^{\exp }$ and Curie-Weiss $\theta^{\exp }$ temperatures are presented as a reference. The superscripts indicate the references from which the experimental values were extracted: $\mathrm{a}=[13], \mathrm{b}=[14]$.

\begin{tabular}{lccr}
\hline \hline & GdCoIn $_{5}$ & GdRhIn $_{5}$ & GdIrIn $_{5}$ \\
\hline$K_{0}$ & 1.31 & 1.21 & 1.51 \\
$K_{1}$ & 1.65 & 1.74 & 1.63 \\
$\boldsymbol{K}_{\mathbf{2}}$ & $\mathbf{0 . 4 7}$ & $\mathbf{1 . 4 3}$ & $\mathbf{1 . 3 0}$ \\
$K_{3}$ & 0.05 & -0.1 & 0.02 \\
$K_{4}$ & -0.11 & -0.15 & -0.12 \\
$\theta$ & 64.2 & 66.5 & 75.2 \\
$\theta^{\exp }$ & $\sim 50$ & $69^{\mathrm{a}}, 63.8^{\mathrm{b}}$ & $64^{\mathrm{a}}$ \\
$T_{N}^{\mathrm{MF}}$ & 44.3 & 57.7 & 52.9 \\
$T_{N}^{\mathrm{QMC}}$ & 32.1 & 41.9 & 38.4 \\
$T_{N}^{\exp }$ & 30 & 39 & 40 \\
\hline \hline
\end{tabular}

$5 d$ conduction electrons with a magnetic exchange coupling $J_{f d}$ that for a related material has been estimated to be $J_{f d} \sim 75 \mathrm{meV}$ (see Ref. [31]). The almost empty $\mathrm{Gd}^{3+} 5 d$ orbitals have a small hybridization with the partially occupied transition metal $d$ orbitals (see Fig. 3). With these ingredients we construct a toy model, to describe the behavior of the RKKY coupling $K_{2}$, with parameters that could be estimated from experimental data. We consider a single effective level to represent the transition metal $d$ orbitals and another for the $\mathrm{Gd}^{3+} 5 d$ orbitals. While the In $5 p$ orbitals contribute to the conduction electron bands, their inclusion in the toy model does not change qualitatively the results and will not be considered here. To calculate the exchange coupling $K_{2}$ we consider two Gd atoms coupled via a single transition metal atom and calculate the energy of the parallel $\left(E_{P}\right)$ and antiparallel

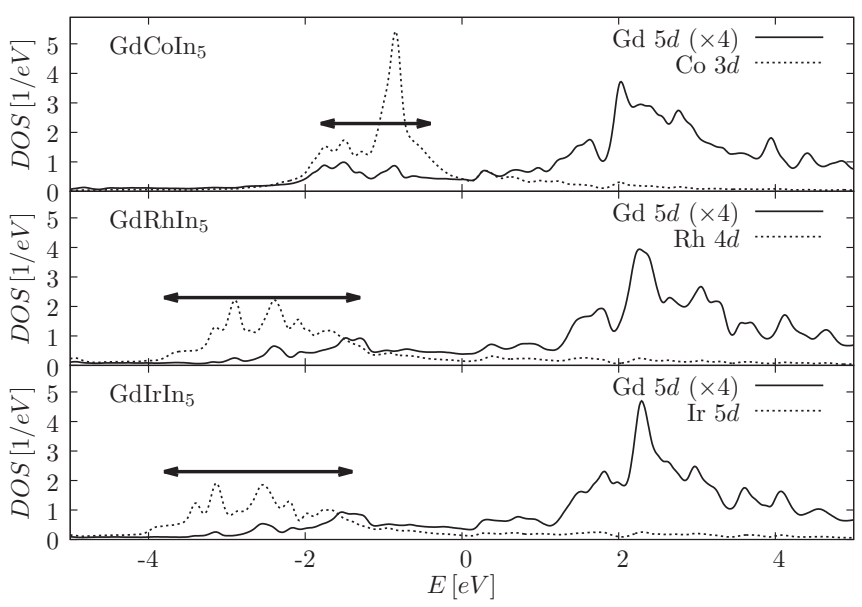

FIG. 3. Partial densities of states of the $\mathrm{Gd}^{3+} 5 d$ and the transition metal $d$ orbital for the three $\operatorname{Gd} M \operatorname{In}_{5}(M=\mathrm{Co}, \mathrm{Rh}, \mathrm{Ir})$ compounds. A small hybridization between the transition metal $M$ and the $\mathrm{Gd}$ electrons can be deduced from the presence of Gd $d$ states at energies where the $M d$ electrons have a sizable DOS. The arrows indicate a rough estimation of the total bandwidth of the bands with mostly $M$ $d$ character. The $\mathrm{Gd}^{3+} 5 d$ partial DOS has been multiplied by 4 .
( $\left.E_{\mathrm{AP}}\right)$ configurations for the $\mathrm{Gd}^{3+} 4 f$ magnetic moments. The coupling is estimated as $K_{2} \sim\left(E_{\mathrm{AP}}-E_{P}\right) / 2 J^{2}$. The model Hamiltonian is

$$
\begin{aligned}
H_{2}= & \sum_{i=1,2} \sum_{\sigma} E_{d_{i \sigma}} d_{i \sigma}^{\dagger} d_{i \sigma}+E_{c} \sum_{\sigma} c_{\sigma}^{\dagger} c_{\sigma} \\
& +t \sum_{i=1,2} \sum_{\sigma}\left(d_{i \sigma}^{\dagger} c_{\sigma}+\text { H.c. }\right),
\end{aligned}
$$

where $d_{i \sigma}^{\dagger}\left(c_{\sigma}^{\dagger}\right)$ creates an electron with spin projection $\sigma= \pm$ along the $z$ axis on the $i$ th $\mathrm{Gd}^{3+} 5 d(M d)$ effective orbital. The exchange coupling between the $4 f$ and $5 d \mathrm{Gd}^{3+}$ electrons is taken as a static field on the $\mathrm{Gd}^{3+} 5 d$ effective orbital making its energy spin dependent: $E_{d \sigma}=E_{d} \pm \sigma \Delta$, where the $+(-)$ sign corresponds to the $\mathrm{Gd}^{3+} 4 f$ magnetic moment being parallel (antiparallel) to the $z$ axis and $\Delta=J J_{f d}$. The model can be readily diagonalized and to lowest order in $t$ and $\Delta$,

$$
K_{2} \sim \frac{2 \Delta^{2} t^{4}}{J^{2}\left(E_{c}-E_{d}\right)^{5}},
$$

where we have assumed that $t$ is a small parameter. The parameters $E_{d}$ and $E_{c}$ can be roughly estimated from the central weights of the bands with the highest $\mathrm{Gd}^{3+} 5 d$ and $M$ $d$ character, respectively. To obtain the ratio of hybridization parameters $t$ for two given compounds, we assumed it to be proportional to the ratio of the total bandwidths of the $M d$ bands in the corresponding compounds (see Fig. 3 for the estimation of the width of the $M d$ bands). The main assumption here is that the intraplane and interplane hybridizations change in the same proportion when the transition metal is changed. The estimation of the parameters from experimental data requires the measurement of the $M d$ and $\mathrm{Gd} d$ partial DOS that could be obtained from resonant photoemission spectroscopy experiments [32].

The parameter $E_{d} \sim 3 \mathrm{eV}$ is nearly the same for the three compounds, while the level energy of the Co $3 d$ orbital $\left(E_{c}^{\mathrm{Co}} \sim-1.1 \mathrm{eV}\right)$ is higher than the corresponding to $\mathrm{Rh} 4 d\left(E_{c}^{\mathrm{Rh}} \sim-2.5 \mathrm{eV}\right)$ and $\mathrm{Ir} 5 d\left(E_{c}^{\mathrm{Ir}} \sim-2.6 \mathrm{eV}\right)$. The hybridization $t$ is estimated (see Fig. 3 ) to be a $\sim 40 \%$ smaller in $\mathrm{GdCoIn}_{5}$ than in the $\mathrm{Rh}$ and $\mathrm{Ir}$ compounds. The model reproduces approximately the value of the ratios between the couplings $K_{2}$ of the three compounds. The reduced value of the $K_{2}$ exchange couplings in the Co-based compound is associated with a reduced hybridization $t$ compared to the $\mathrm{Rh}$ and Ir compounds, which is partially compensated for the larger value of $E_{c}$ in $\mathrm{GdCoIn}_{5}$. The coupling $K_{2}$ is expected to have similar values for $\mathrm{GdRhIn}_{5}$ and $\mathrm{GdIrIn}_{5}$ as the two materials have similar effective parameters and hybridization $t$. Similar results are obtained estimating the parameters from a Wannier orbital analysis projecting the Hamiltonian on the partially occupied $\mathrm{Gd}^{3+} 5 d$ and $M d$ bands [19].

\section{Finite temperatures}

In this section we analyze the validity of the model Hamiltonian given by Eq. (1) and the calculated exchange coupling parameters (see Table IV) to describe the magnetic degrees of freedom in the $\mathrm{Gd} M \mathrm{In}_{5}$ compounds. We solve the magnetic Hamiltonian using different approximations to obtain the magnetic susceptibility and the magnetic 


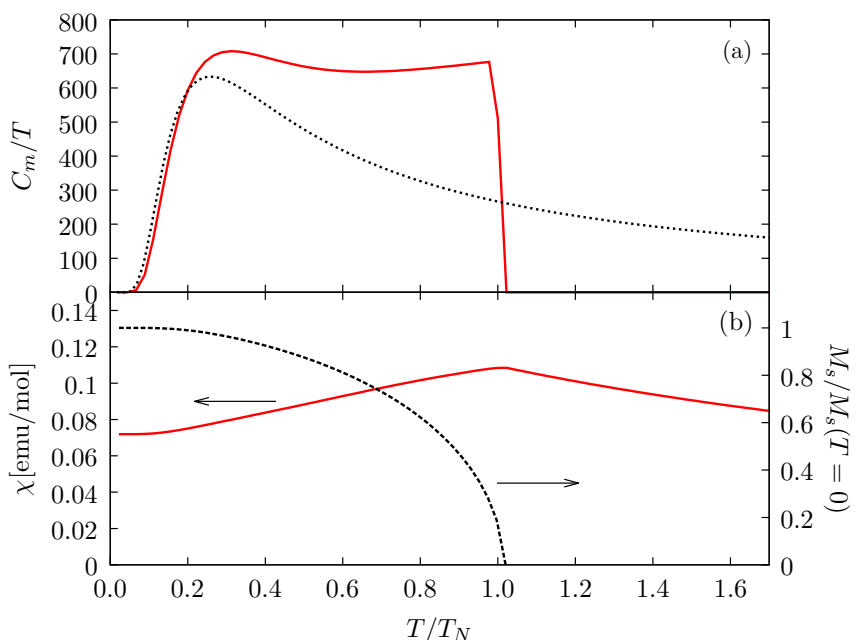

FIG. 4. (Color online) Mean-field solution of the magnetic Hamiltonian of Eq. (1) using the calculated parameters for $\mathrm{GdCoIn}_{5}$ from Table IV. (a) Specific heat $C_{m} / T$ as a function of the temperature calculated using the coupling parameters for $\mathrm{GdCoIn}_{5}$ (solid line) and for a two-level system with level splitting $2 k_{B} T_{N} / 3$ (dotted line). (b) Crystal averaged magnetic susceptibility (left axis) and staggered magnetization (right axis).

contribution to the specific heat and compare with the experimental data in the literature. We solved the magnetic Hamiltonian given by Eq. (1) in the mean-field (MF) approximation considering eight independent $\mathrm{Gd}^{3+}$ magnetic moments. The model presents a paramagnetic to $C$-type antiferromagnet transition as the temperature is lowered below $T_{N}^{\mathrm{MF}}=\frac{J(J+1)}{3}\left(4 K_{1}+2 K_{2}-8 K_{4}\right)$. Figure 4(a) presents the magnetic contribution to the specific heat which shows a discontinuity at the transition temperature and vanishes above $T_{N}$. The obtained behavior of $C_{m}$ for temperatures above $T_{N}$ is a well-known artifact of the MF solution. The shoulder in $C_{m} / T$ below the transition temperature is due to an increase in the staggered magnetization and the associated internal field as the temperature is lowered. The coupling to the internal field splits the different projections of each magnetic moment along the internal field axis and the higher energy projections are exponentially suppressed as the temperature is lowered. The peak in $C_{m} / T$ at $T \sim 0.3 T_{N}$ can be associated with a Schotkky-like anomaly as $k_{B} T$ becomes of the order of the energy splitting $\Delta(T)$ between the two lowest-lying states. This is illustrated by the specific heat contribution for a two-level system with temperature-independent energy splitting $\Delta(T=0)=\frac{2}{3} k_{B} T_{N}$ shown in Fig. 4(a) [33]. The second-order transition at $T_{N}$ is accompanied by the onset of the staggered magnetization $M_{s}$ [see Fig. 4(b)] associated with an internal field $\mu_{\mathrm{B}} H_{\mathrm{int}}=\frac{3 k_{\mathrm{B}} T_{N}^{\mathrm{MF}}}{J(J+1)}\langle\mathcal{J}\rangle$. As $T \rightarrow 0$ the staggered magnetization saturates and the specific heat is exponentially reduced at the MF level.

The magnetic susceptibility is presented in Fig. 4(b). At temperatures $T>T_{N}, \chi$ has a Curie-Weiss behavior and decreases with decreasing temperature: $\chi=\left(g_{\mathrm{J}} \mu_{\mathrm{B}}\right)^{2} J(J+1) /$ $3(T+\theta)$, where $\theta=\frac{J(J+1)}{3}\left(4 K_{0}+4 K_{1}+2 K_{2}+8 K_{3}+\right.$ $\left.8 K_{4}\right)$. The values of $\theta$ for the $\mathrm{Gd} M \mathrm{In}_{5}$ compounds using the calculated exchange couplings are presented in Table IV.

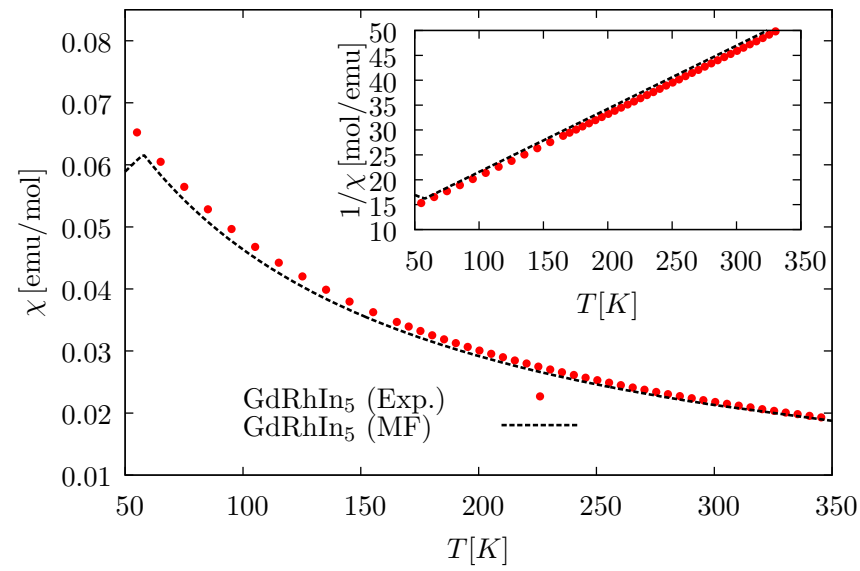

FIG. 5. (Color online) Crystal averaged magnetic susceptibility $\chi=M / B, B=0.1 T$. Mean-field and experimental [13] results for $\mathrm{GdRhIn}_{5}$. (Inset) Inverse magnetic susceptibility as a function of the temperature showing a Curie-Weiss behavior for $T>T_{N}$. The MF results provide an accurate description at high temperatures.

They present a good agreement with the experimental results. Figure 5 presents a comparison between the MF and the experimental results for the magnetic susceptibility of $\mathrm{GdRhIn}_{5}$ as a function of the temperature. There is an excellent agreement at temperatures above the Néel temperature where the material presents a Curie-Weiss behavior (see inset to Fig. 5). The Néel temperature is overestimated, which, as we see below, is a consequence of ignoring quantum fluctuations and the source of the low-temperature disagreement between the MF magnetic susceptibility and the experimental data.

Although the MF solution is consistent with the experimental results, it does not show some features in the specific heat like the power-law behavior of $C / T$ at small $T$ (related with spin waves) nor the $\lambda$ divergence at the transition and overestimates the transition temperature. To improve the description of the physical properties including quantum fluctuations, we resort to a simplified magnetic model. We consider $J=7 / 2$ magnetic moments on a cubic lattice interacting through a first-neighbor antiferromagnetic exchange coupling $K_{\text {eff }}=\frac{3 k_{\mathrm{B}} T_{N}^{\mathrm{MF}}}{J(J+1)} / z$, where $z=6$ is the number of neighbors. At the MF level the simplified model reproduces the transition temperature and the specific heat of the full model in the complete temperature range. It does not reproduce, however, the Curie-Weiss temperature, which, within the MF approximation, is now equal to $-T_{N}$. The effective model can be solved numerically using quantum Monte Carlo simulations in a finite size cluster. We considered sizes ranging from $L=6$ up to $L=30$ in a cubic lattice of $L \times L \times L$ sites and used a finite size scaling analysis to extrapolate to $L \rightarrow \infty$. Thermalization and measurements were performed with a temperature-dependent number of sweep steps, ranging between $10^{5}$ and $10^{6}$ steps. The quantum Monte Carlo simulations were performed using the ALPS [34] library, in particular, the "loop" algorithm, which allows the inclusion of external magnetic fields.

The results for the magnetic contribution to the specific heat are presented in Fig. 6 together with the experimental 


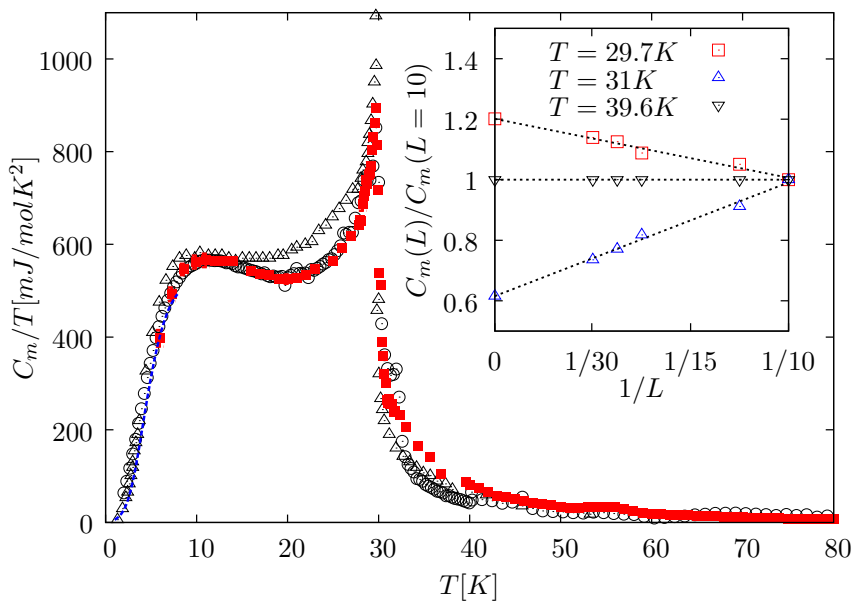

FIG. 6. (Color online) Magnetic contribution to the specific heat in $\mathrm{GdCoIn}_{5}$ and $\mathrm{GdRhIn}_{5}$. Experiment: $\mathrm{GdCoIn}_{5}$ (open circles) and $\mathrm{GdRhIn}_{5}$ (open triangles). Theory: quantum Monte Carlo (red solid squares). The experimental data for $\mathrm{GdCoIn}_{5}$ was obtained subtracting the calculated electron and phonon contributions to the specific heat. The experimental values for $\mathrm{GdRhIn}_{5}$ were extracted from Ref. [14] and rescaled to ease the comparison with the $\mathrm{GdCoIn}_{5}$ data: $T \rightarrow \zeta T, C_{m} / T \rightarrow \zeta^{-1} C_{m} / T$, where $\zeta=$ Néel temperature of $\mathrm{GdCoIn}_{5} /$ Néel temperature of GdRhIn ${ }_{5}$. The blue dashed line is the result of a spin-wave calculation valid at low temperatures. (Inset) Finite size scaling of the quantum Monte Carlo results for the specific heat. The lines are linear fits from which the $L \rightarrow \infty$ limit of the specific heat values were extracted.

data [8]. The experimental $C_{m}$ for $\mathrm{GdCoIn}_{5}$ was obtained subtracting the theoretically obtained electronic and phonon contributions [35]. The phonon contribution was corrected to account for anharmonic effects (see Refs. [8] and [36]). We used a slightly reduced coupling $0.93 K_{\text {eff }}$ in order to match the experimental transition temperature of $\mathrm{GdCoIn}_{5}$. For a given coupling strength, the MF solution overestimates the transition temperature by $\sim 50 \%$ [37]. The experimental data for $\mathrm{GdRhIn}_{5}$ was extracted from Ref. [14], where the magnetic contribution to the specific heat was obtained subtracting the specific heat of the nonmagnetic YRhIn 5 . The QMC results based on the DFT calculated magnetic interaction parameters predict a transition temperature within a $10 \%$ of the experimental observation for the three $\mathrm{GdMIn}_{5}$ compounds [37]. An excellent experiment-theory agreement is obtained in the full temperature range of $C_{m} / T$, including the high-temperature tail, the $\lambda$ transition, the plateau, and the Schotkky-like anomaly. For temperatures close to $T_{N}$ the finite size effects are maximal and are corrected using a finite size scaling (see inset to Fig. 6). At low temperatures $(T \lesssim 10 \mathrm{~K})$ the error in the numerical calculations increases. To complete the description, we perform a spin-wave analysis. The antiferromagnetic spin waves in a cubic lattice have a dispersion relation

$$
\omega(\mathbf{q})=2 K_{\text {eff }} J \sqrt{9-\left(\cos q_{x}+\cos q_{y}+\cos q_{z}\right)^{2}},
$$

and the specific heat is

$$
C_{s w}(T)=R \frac{\partial}{\partial T} \int_{0}^{\infty} \omega(\mathbf{q}) n_{b}[\omega(\mathbf{q})] d^{3} q,
$$

where $n_{b}(\omega)$ is the Bose-Einstein distribution and $R$ is the universal gas constant. In the $T \rightarrow 0$ limit we have the expected temperature behavior $C_{s w}(T \rightarrow 0)=R \frac{32 \pi^{5}}{15(2 z)^{3 / 2}}\left(\frac{T}{J K_{\text {eff }}}\right)^{3}$. The resulting $C_{s w}$ allows us to extend the QMC results to low temperatures and is presented with a blue dashed line in Fig. 6.

\section{SUMMARY AND CONCLUSIONS}

We analyzed the role of the transition metal in the $R M \mathrm{In}_{5}$ family of compounds. We focused our analysis on the $\operatorname{Gd} M \mathrm{In}_{5}(M=\mathrm{Co}, \mathrm{Rh}, \mathrm{Ir})$, which order magnetically at low temperatures. Based on DFT calculations we obtained the parameters of a magnetic Hamiltonian that we solved in the MF approximation and numerically through quantum Monte Carlo calculations. We obtained an excellent agreement with the experimental transition and the Curie-Weiss temperatures, as well as with the specific heat in the full temperature range. Our results show that the source of the diminished Néel temperature observed in the Co-based 115 compounds, compared to the $\mathrm{Rh}$ or Ir-based compounds is associated with a reduced exchange coupling between the magnetic moments of the $\mathrm{Gd}^{3+}$ ions located on different $\mathrm{GdIn}_{3}$ planes. This reduced interplane RKKY coupling is associated with a reduced hybridization between the Gd $5 d$ and the Co $3 d$ electrons. We believe that the reduced coupling between planes may be the source of reduced $T_{N}$ on other compounds of the series. Our results indicate that the magnetism in the Co-based materials has a more two-dimensional character than in their $\mathrm{Rh}$ and $\mathrm{Ir}$ counterparts. This behavior may also help understand the larger superconducting transition temperatures observed in the Co-based Ce-115 and Pu-115 compounds, compared with the Rh- or Ir-based counterparts.

Future work includes extending our theoretical and experimental analysis to the Tb-115 compounds that present sizable crystal-field effects.

\section{ACKNOWLEDGMENTS}

We thank E. Granado, D. Khomskii, P. Pagliuso, R. Lora Serrano, and J. Sofo for stimulating discussions and providing experimental data for the $\mathrm{GdCoIn}_{5}$ and $\mathrm{GdRhIn}_{5}$ compounds. We acknowledge financial support from CONICET Grants No. PIP00832, No. PIP00273, and No. PIP0702, ANPCyT Grant No. PICT07-00812, and SeCTyP-UNCuyo Grant No. 06/C393.
[1] C. Petrovic, P. G. Pagliuso, M. F. Hundley, R. Movshovich, J. L. Sarrao, J. D. Thompson, Z. Fisk, and P. Monthoux, J. Phys: Condens. Matter 13, L337 (2001).
[2] R. Movshovich, M. Jaime, J. D. Thompson, C. Petrovic, Z. Fisk, P. G. Pagliuso, and J. L. Sarrao, Phys. Rev. Lett. 86, 5152 (2001). 
[3] T. Park, F. Ronning, H. Q. Yuan, M. B. Salamon, R. Movshovich, J. Sarrao, and J. D. Thompson, Nature (London) 440, 65 (2006).

[4] E. D. Bauer, M. M. Altarawneh, P. H. Tobash, K. Gofryk, O. E. Ayala-Valenzuela, J. N. Mitchell, R. D. McDonald, C. H. Mielke, F. Ronning, J.-C. Griveau, E. Colineau, R. Eloirdi, R. Caciuffo, B. L. Scott, O. Janka, S. M. Kauzlarich, and J. D. Thompson, J. Phys: Condens. Matter 24, 052206 (2012).

[5] E. Bauer et al. (unpublished).

[6] J. Hudis, R. Hu, C. Broholm, V. Mitrović, and C. Petrovic, J. Magn. Magn. Mater. 307, 301 (2006).

[7] M. Koeda, M. Hedo, T. Fujiwara, Y. Uwatoko, T. Sadamasa, and Y. Inada, J. Phys. Soc. Jpn. 76, 62 (2007).

[8] D. Betancourth, J. Facio, P. Pedrazzini, C. Jesus, P. Pagliuso, V. Vildosola, P. S. Cornaglia, D. García, and V. Correa, J. Magn. Magn. Mater. 374, 744 (2015).

[9] Y. Isikawa, D. Kato, A. Mitsuda, T. Mizushima, and T. Kuwai, J. Magn. Magn. Mater. 272-276, 635 (2004).

[10] H. T. Huy, S. Noguchi, N. V. Hieu, X. Shao, T. Sugimoto, and T. Ishida, J. Magn. Magn. Mater. 321, 2425 (2009).

[11] H. Hegger, C. Petrovic, E. G. Moshopoulou, M. F. Hundley, J. L. Sarrao, Z. Fisk, and J. D. Thompson, Phys. Rev. Lett. 84, 4986 (2000).

[12] P. G. Pagliuso, J. D. Thompson, M. F. Hundley, and J. L. Sarrao, Phys. Rev. B 62, 12266 (2000).

[13] P. G. Pagliuso, J. D. Thompson, M. F. Hundley, J. L. Sarrao, and Z. Fisk, Phys. Rev. B 63, 054426 (2001).

[14] N. Van Hieu, T. Takeuchi, H. Shishido, C. Tonohiro, T. Yamada, H. Nakashima, K. Sugiyama, R. Settai, T. D. Matsuda, Y. Haga, M. Hagiwara, K. Kindo, S. Araki, Y. Nozue, and Y. Onuki, J. Phys. Soc. Jpn. 76, 064702 (2007).

[15] R. Lora-Serrano, C. Giles, E. Granado, D. J. Garcia, E. Miranda, O. Agüero, L. Mendonça Ferreira, J. G. S. Duque, and P. G. Pagliuso, Phys. Rev. B 74, 214404 (2006).

[16] N. Van Hieu, H. Shishido, T. Takeuchi, A. Thamizhavel, H. Nakashima, K. Sugiyama, R. Settai, T. D. Matsuda, Y. Haga, M. Hagiwara, K. Kindo, and Y. Onuki, J. Phys. Soc. Jpn. 75, 074708 (2006).

[17] C. Petrovic, R. Movshovich, M. Jaime, P. G. Pagliuso, M. F. Hundley, J. L. Sarrao, Z. Fisk, and J. D. Thompson, Europhys. Lett. 53, 354 (2001).

[18] R. L. Serrano, Determinaçao de estruturas magnéticas de novos compostos intermetálicos (Biblioteca Digital da Unicamp, 2006).
[19] V. I. Anisimov, D. E. Kondakov, A. V. Kozhevnikov, I. A. Nekrasov, Z. V. Pchelkina, J. W. Allen, S.-K. Mo, H.-D. Kim, P. Metcalf, S. Suga, A. Sekiyama, G. Keller, I. Leonov, X. Ren, and D. Vollhardt, Phys. Rev. B 71, 125119 (2005).

[20] P. Blaha, K. Schwarz, G. K. H. Madsen, D. Kvasnicka, and J. Luitz, WIEN2K, An Augmented Plane Wave + Local Orbitals Program for Calculating Crystal Properties (Karlheinz Schwarz, Technische Universität Wien, Austria, 2001).

[21] J. P. Perdew, K. Burke, and M. Ernzerhof, Phys. Rev. Lett. 77, 3865 (1996).

[22] P. Piekarz, K. Parlinski, P. T. Jochym, A. M. Oleś, J.-P. Sanchez, and J. Rebizant, Phys. Rev. B 72, 014521 (2005).

[23] S. Raymond, P. Piekarz, J. P. Sanchez, J. Serrano, M. Krisch, B. Janoušová, J. Rebizant, N. Metoki, K. Kaneko, P. T. Jochym, A. M. Oleś, and K. Parlinski, Phys. Rev. Lett. 96, 237003 (2006).

[24] J.-X. Zhu, P. H. Tobash, E. D. Bauer, F. Ronning, B. L. Scott, K. Haule, G. Kotliar, R. C. Albers, and J. M. Wills, Europhys. Lett. 97, 57001 (2012).

[25] V. I. Anisimov, I. V. Solovyev, M. A. Korotin, M. T. Czyżyk, and G. A. Sawatzky, Phys. Rev. B 48, 16929 (1993).

[26] Z. P. Yin and W. E. Pickett, Phys. Rev. B 74, 205106 (2006).

[27] M. Petersen, J. Hafner, and M. Marsman, J. Phys.: Condens. Matter 18, 7021 (2006).

[28] E. Granado, B. Uchoa, A. Malachias, R. Lora-Serrano, P. G. Pagliuso, and H. Westfahl, Jr., Phys. Rev. B 74, 214428 (2006).

[29] S. Chang, P. G. Pagliuso, W. Bao, J. S. Gardner, I. P. Swainson, J. L. Sarrao, and H. Nakotte, Phys. Rev. B 66, 132417 (2002).

[30] J. Jensen and A. R. Mackintosh, Rare Earth Magnetism (Clarendon Press, Oxford, UK, 1991).

[31] M. Cabrera-Baez, W. Iwamoto, E. T. Magnavita, J. M. OsorioGuillén, R. a. Ribeiro, M. a. Avila, and C. Rettori, J. Phys.: Condens. Matter 26, 175501 (2014).

[32] G. Levy, R. Sutarto, D. Chevrier, T. Regier, R. Blyth, J. Geck, S. Wurmehl, L. Harnagea, H. Wadati, T. Mizokawa, I. S. Elfimov, A. Damascelli, and G. A. Sawatzky, Phys. Rev. Lett. 109, 077001 (2012).

[33] J. Blanco, D. Gignoux, and D. Schmitt, Phys. Rev. B 43, 13145 (1991).

[34] A. W. Sandvik, Phys. Rev. B 59, R14157 (1999).

[35] A detailed analysis of the phonon contribution to the specific heat in these compounds will be presented elsewhere.

[36] D. Wallace, Thermodynamics of Crystals (Dover, Mineola, NY, 1998).

[37] J. Oitmaa and W. Zheng, J. Phys: Condens. Matter 16, 8653 (2004). 